\title{
INVOLUTIONS IN CONWAY'S LARGEST SIMPLE GROUP
}

\section{CHRIS BATES AND PETER ROWLEY}

\begin{abstract}
In this paper, the authors determine the suborbits of Conway's largest simple group in its conjugation action on each of its three conjugacy classes of involutions. Matrix representatives of these suborbits are also provided in an accompanying computer file.
\end{abstract}

\section{Introduction}

The tentacles of Conway's largest simple group, $\mathrm{Co}_{1}$, reach out in a number of different directions. Through its connections with the Leech lattice, $\Lambda$, it interacts with sphere packings and related combinatorial matters (see [8]). Via the rich structure inherent in the Leech lattice, we also see many of the fellow sporadic simple groups of $\mathrm{Co}_{1}$ as subgroups; see, for example, [6, Table 5] and the ATLAs [7] for the stabilizers of various sets of vectors in $\Lambda$. This group may also be viewed as a 'stepping stone' en route to the Monster simple group; consult [5] and [12] for more on this. The subgroup structure of $\mathrm{Co}_{1}$ has received much attention. Wilson [15] has completed the enumeration of its maximal subgroups, following on from earlier work of Curtis in [9] and [10]. Yet there is still much more to be discovered about this group. As one example, we mention the speculative investigations of Harada and Lang [13], which may be pointing to new horizons.

From now on, we shall let $G$ denote $\mathrm{Co}_{1}$, and we let $t$ denote a fixed involution of $G$. There are three $G$-conjugacy classes of involutions, namely $2 A, 2 B$ and $2 C$ (we use the class names as given in the ATLAs [7]). In this paper, we determine the suborbits of $G$ in its (conjugation) action on $t^{G}$, together with various other related information. So we are looking for the $C_{G}(t)$-orbits of $t^{G}$. We recall the facts shown in Table 1 (see [7] and [15]).

The permutation ranks may be verified using GAP [14]. (In [4], the permutation character $1_{C_{G}(t)}^{G}$ is given for $t$ in $2 A, 2 B$ and $2 C$.) A study of the commuting involution graph for $\mathrm{Co}_{1}$, currently being undertaken by Bundy, Perkins, and the authors, has prompted some of the calculations that we describe here. It is anticipated that the results presented here will be of use in other investigations.

In Section 2 we give the tabular information concerning the size of suborbits, while Appendix A contains other data that sometimes allows faster identification of which suborbit an involution is in. Finally, in Appendix B we provide lists of representatives for each

Table 1: Permutation rank.

\begin{tabular}{c|cc}
\hline$t^{G}$ & $C_{G}(t)$ & Permutation Rank on $t^{G}$ \\
\hline $2 A$ & $2_{+}^{1+8} \cdot O_{8}^{+}(2)$ & 11 \\
$2 B$ & $\left(2^{2} \times G_{2}(4)\right): 2$ & 59 \\
$2 C$ & $2^{11}: M_{12}: 2$ & 177 \\
\hline
\end{tabular}

Received 21 November 2003, revised 2 November 2004; published 23 December 2004. 
of the suborbits (as $24 \times 24$ matrices over $G F(2)$ ) in GAP [14], Magma [2] and MeatAxe formats. For further details, see Section 2.4. We note here that for all the computational work involved in determining the suborbits, we use the 24-dimensional representation over $G F(2)$ given in [16]. So we start with the standard generators for $\mathrm{Co}_{1}, a$ and $b$, where $a$ is in class $2 B, b$ is in class $3 C, a b$ has order 40 and $a b a b b$ has order 6 . In the file we also outline a routine for obtaining any of the given $C_{G}(t)$-orbit representatives as expressions in the standard generators $a$ and $b$.

\section{The suborbits of $t^{G}$}

Let $t$ be a fixed involution in $G$, and set $X=t^{G}$. Let $x \in X$, and suppose that $C$ is a $G$-conjugacy class. The set $X_{C}:=\{x \in X: t x \in C\}$ clearly breaks up into orbits under the conjugation action of $C_{G}(t)$; so if we wish to determine all the suborbits of $t^{G}$, we need to find the $C_{G}(t)$-orbits of $X_{C}$, as $C$ ranges over all those $C$ for which $X_{C}$ is non-empty.

In order to examine the possibilities for $C$ and the cardinality of the corresponding set $X_{C}$, we use the method of class structure constants. These describe the number of possible products of elements from a conjugacy class $C_{j}$ with elements from a conjugacy class $C_{l}$ that give an element in some conjugacy class $C_{i}$ of $G$. In the current situation, we have $X=C_{j}=C_{l}$ and $C=C_{i}$, in which case the class structure constant is equal to

$$
\xi_{i}=\frac{|G|}{\left|C_{G}(t)\right|^{2}} \sum_{r=1}^{k} \frac{\chi_{r}^{2}(t) \overline{\chi\left(g_{i}\right)}}{\chi_{r}(1)}
$$

where $g_{i}$ is a representative of the class $C_{i}, k$ is the number of conjugacy classes in $G$, and the $\chi_{r}$ are the irreducible complex characters of $G$ (see, for example, [11]). We can thus calculate these constants directly from the complex character tables of the $G$, which are recorded in the ATLAS [7], and are available electronically in the standard libraries of the computer algebra package GAP [14] .

During our computations, we need to identify the conjugacy class of various elements. To do this, we use class invariants such as order, dimension of the fixed space, and centralizer size, as well as the various power maps. This information is drawn primarily from the ATLAS [7]. In cases where we need to compute the centralizer of an element, we can sometimes employ the procedure introduced in [1] to allow for faster identification. We note here that we use the method described in [3] to generate involution centralizers.

We are often faced with the problem of finding $x \in X$ such that $z=t x$ belongs to a given class $C$. If $\left|C_{G}(z)\right|$ is large, these elements are rare, and so a basic search is unlikely to yield results. We overcome this problem by searching for elements of a higher order. In other words, we look for $y \in X$ such that $(t y)^{m}$ lies in $C$, for some $m \in \mathbb{N}$. Then $t(t y)^{m} \in X$ is an appropriate element.

Given $x, y \in X$ such that $t x$ and $t y$ belong to the same $G$-conjugacy class, it is computationally expensive to determine whether or not they are conjugate under $C_{G}(t)$ by using the standard algorithm implemented in the computer algebra package MAGMA. This can prove to be a problem, especially when the orbit sizes are small, and so we try to exploit the structure of $C_{G}(t)$ in order to identify distinct orbits more efficiently. We shall give specific details of this for $t \in 2 A, t \in 2 B$ and $t \in 2 C$ in Sections 2.1,2.2 and 2.3, respectively. An additional way of trying to determine whether $x$ and $y$ lie in different $C_{G}(t)$-orbits is to calculate the dimensions of the subspaces fixed by the subgroups $\langle t, x\rangle$ and $\langle t, y\rangle$, and to observe any differences. As a last resort, we do in fact call upon the 'IsConjugate $\left(C_{G}(t), t x, t y\right)$ ' command in MAGMA. 
In the course of our calculations, we often needed to identify the conjugacy class to which an element $g$ of $\mathrm{Co}_{1}$ belongs. This was usually achieved by looking at the various powers of $g$, and determining $\operatorname{dim}\left(C_{V}(g)\right)$ (where $V$ is the 24-dimensional $G F(2) \mathrm{Co}_{1}$-module given by [16]). Since this data may be of value elsewhere, it is given as Appendix A of this paper.

\section{1. $t \in 2 A$}

In this case, the permutation rank of $G$ on $X=t^{G}$ is only 11 , and it is relatively easy to determine the suborbits. We include them here for completeness. Recall that $C_{G}(t) \cong$ $2_{+}^{1+8} \cdot O_{8}^{+}(2)$ and $N=O_{2}\left(C_{G}(t)\right) \cong 2_{+}^{1+8}$. There are four $C_{G}(t)$-conjugacy classes of $N$, two of which are of size one, consisting only of the identity or $t$. The other two, $C_{1}$ and $C_{2}$, have cardinalities of 270 and 240, respectively. (In fact, $C_{1}$ is contained in $2 A$, and $C_{2}$ is contained in $4 A$.) For each representative $x \in X$, we calculate the suborbit invariants $c_{1}=\left|C_{N}(x) \cap C_{1}\right|$ and $c_{2}=\left|C_{N}(x) \cap C_{2}\right|$. These are listed in Table 2, along with $|\mathcal{O}|$ (the size of each suborbit) and $d$ (the dimension of the subspace fixed by $\langle t, x\rangle$ ).

\section{2. $t \in 2 B$}

Taking $t \in 2 B$, in this case we have $C_{G}(t) \cong\left(2^{2} \times G_{2}(4)\right): 2$. Let $L \unlhd \unlhd C_{G}(t)$ be such that $L \cong G_{2}(4)$. Then $D=L C_{C_{G}(t)}(L)=F \times L \cong 2^{2} \times G_{2}(4)$. Now let $x \in X=t^{G}$, and suppose that $z=t x$ has even order $l$, for some $l \in \mathbb{N}$. Then $\langle t, x\rangle$ is a dihedral group of order $2 l$, with centre generated by $(t x)^{l / 2}$. In particular, the involution $(t x)^{l / 2}$ lies in $C_{G}(t)$. As a way of trying to distinguish such $x$ from other suborbits, we can determine whether $(t x)^{l / 2}$ can be found in $F$, in $L$ or as a diagonal element in $D$, or whether it lies outside of $D$. This information is listed in Table 3 , in the last column, which is labelled $\mathcal{L}$ (for location); we use, $L, F, D$ and $O$ to mean that $(t x)^{l / 2}$ is in, respectively, $L, F, D \backslash(L \cup F)$ and $C_{G}(t) \backslash D$. Also, in the column labelled $(t x)^{l / 2}$, we give the class of $(t x)^{l / 2}$. As usual, for every orbit representative $x$, we calculate $d$, the dimension of the subspace fixed by $\langle t, x\rangle$, and we sometimes use it as a tool for distinguishing orbits.

We note that the $\mathcal{L}$ data sometimes puts daylight between otherwise similar $C_{G}(t)$-orbits when $t x \in 4 E \cup 12 L$.

Table 2: $t \in 2 A$.

\begin{tabular}{c|rrrr}
\hline$C$ & $|\mathcal{O}|$ & $d$ & $c_{1}$ & \multicolumn{1}{c}{$c_{2}$} \\
\hline $2 A$ & 12600 & 12 & 78 & 48 \\
& 270 & 12 & 142 & 112 \\
$2 C$ & 60480 & 10 & 30 & 0 \\
$3 B$ & 573440 & 10 & 0 & 0 \\
$3 D$ & 491520 & 8 & 0 & 0 \\
$4 A$ & 34560 & 8 & 1 & 0 \\
$4 C$ & 4838400 & 9 & 3 & 0 \\
$4 D$ & 2419200 & 8 & 1 & 0 \\
$5 B$ & 12386304 & 8 & 0 & 0 \\
$6 E$ & 25804800 & 8 & 0 & 0 \\
\hline
\end{tabular}


Table 3: $t \in 2 B$.

\begin{tabular}{|c|c|c|c|c|}
\hline$C$ & $|\mathcal{O}|$ & $d$ & $(t x)^{l / 2}$ & $\mathcal{L}$ \\
\hline $2 A$ & 4095 & 8 & $2 A$ & $L$ \\
\hline \multirow[t]{3}{*}{$2 B$} & 41600 & 6 & $2 B$ & $O$ \\
\hline & 8190 & 8 & $2 B$ & $D$ \\
\hline & 2 & 12 & $2 B$ & $F$ \\
\hline $2 C$ & 65520 & 6 & $2 C$ & $D$ \\
\hline $3 A$ & 1664 & 0 & - & - \\
\hline $3 B$ & 166400 & 6 & - & - \\
\hline $3 D$ & 4193280 & 4 & - & - \\
\hline \multirow[t]{2}{*}{$4 A$} & 81900 & 4 & $2 A$ & $L$ \\
\hline & 16380 & 4 & $2 A$ & $L$ \\
\hline \multirow[t]{2}{*}{$4 B$} & 327600 & 4 & $2 A$ & $L$ \\
\hline & 163800 & 4 & $2 A$ & $L$ \\
\hline \multirow[t]{2}{*}{$4 D$} & 2620800 & 4 & $2 A$ & $L$ \\
\hline & 655200 & 4 & $2 A$ & $L$ \\
\hline \multirow[t]{4}{*}{$4 E$} & 5241600 & 4 & $2 B$ & $O$ \\
\hline & 5241600 & 4 & $2 B$ & $D$ \\
\hline & 83200 & 6 & $2 B$ & $F$ \\
\hline & 83200 & 6 & $2 B$ & $O$ \\
\hline \multirow[t]{2}{*}{$5 A$} & 1048320 & 0 & - & - \\
\hline & 3328 & 0 & - & - \\
\hline $5 B$ & 8386560 & 4 & - & - \\
\hline $6 A$ & 524160 & 0 & $2 A$ & $L$ \\
\hline $6 B$ & 2995200 & 0 & $2 B$ & $O$ \\
\hline $6 E$ & 10483200 & 4 & $2 A$ & $L$ \\
\hline \multirow[t]{2}{*}{$6 H$} & 83865600 & 2 & $2 B$ & $O$ \\
\hline & 8386560 & 4 & $2 B$ & $D$ \\
\hline $6 I$ & 62899200 & 2 & $2 C$ & $D$ \\
\hline $7 A$ & 16773120 & 0 & - & - \\
\hline
\end{tabular}

\begin{tabular}{|c|c|c|c|c|}
\hline$C$ & $|\mathcal{O}|$ & $d$ & $(t x)^{l / 2}$ & $\mathcal{L}$ \\
\hline \multirow[t]{2}{*}{$8 A$} & 2620800 & 2 & $2 A$ & $L$ \\
\hline & 2620800 & 2 & $2 A$ & $L$ \\
\hline \multirow[t]{2}{*}{$8 B$} & 7862400 & 2 & $2 A$ & $L$ \\
\hline & 7862400 & 2 & $2 A$ & $L$ \\
\hline \multirow[t]{3}{*}{$8 C$} & 10483200 & 2 & $2 A$ & $L$ \\
\hline & 5241600 & 2 & $2 A$ & $L$ \\
\hline & 5241600 & 2 & $2 A$ & $L$ \\
\hline \multirow[t]{2}{*}{$8 F$} & 20966400 & 2 & $2 A$ & $L$ \\
\hline & 20966400 & 2 & $2 A$ & $L$ \\
\hline \multirow[t]{3}{*}{$10 A$} & 10483200 & 0 & $2 A$ & $L$ \\
\hline & 1048320 & 0 & $2 A$ & $L$ \\
\hline & 1048320 & 0 & $2 A$ & $L$ \\
\hline \multirow[t]{2}{*}{$10 C$} & 8386560 & 4 & $2 B$ & $D$ \\
\hline & 8386560 & 4 & $2 B$ & $D$ \\
\hline $10 F$ & 125798400 & 2 & $2 C$ & $D$ \\
\hline $11 A$ & 335462400 & 2 & - & - \\
\hline \multirow[t]{2}{*}{$12 A$} & 5241600 & 0 & $2 A$ & $L$ \\
\hline & 1048320 & 0 & $2 A$ & $L$ \\
\hline $12 C$ & 20966400 & 0 & $2 A$ & $L$ \\
\hline $12 E$ & 41932800 & 2 & $2 A$ & $L$ \\
\hline \multirow[t]{2}{*}{$12 L$} & 167731200 & 2 & $2 B$ & $O$ \\
\hline & 167731200 & 2 & $2 B$ & $D$ \\
\hline $13 A$ & 167731200 & 0 & - & - \\
\hline $14 A$ & 167731200 & 0 & $2 B$ & $O$ \\
\hline $15 B$ & 33546240 & 0 & - & - \\
\hline $15 C$ & 167731200 & 0 & - & - \\
\hline $20 A$ & 83865600 & 0 & $2 A$ & $L$ \\
\hline $21 A$ & 167731200 & 0 & - & - \\
\hline \multirow[t]{2}{*}{$24 A$} & 41932800 & 0 & $2 A$ & $L$ \\
\hline & 41932800 & 0 & $2 A$ & $L$ \\
\hline
\end{tabular}




\section{3. $t \in 2 C$}

When $t \in 2 C$, the permutation rank of $G$ on $X=t^{G}$ is 177 , and there are 87 non-zero structure constants, so determining the suborbits requires a substantial amount of work. Put $N=O_{2}\left(C_{G}(t)\right)$. In $N$ there are six $C_{G}(t)$-conjugacy classes, two of which have size one, just as in Section 2.1. We denote the four classes of size greater than one by $C_{1}, C_{2}, C_{3}$ and $C_{4}$, and we remark that $C_{1}$ and $C_{2}$ are contained in $2 C$, while $C_{3}$ and $C_{4}$ are contained in $2 A$. For $x \in X$ and $i \in\{1, \ldots 4\}$, we calculate the suborbit invariants $c_{i}=\left|C_{N}(x) \cap C_{i}\right|$. As usual, these often provide a useful way of identifying distinct suborbits without having to resort to more computationally intensive methods. These are listed in Table 4, along with the dimension of the subspace fixed by $\langle t, x\rangle$ and the size $|\mathcal{O}|$ of each suborbit.

As we observe in Table 4, the $c_{i}$ data may in some instances serve to distinguish between $C_{G}(t)$-orbits that have the same $|\mathcal{O}|, d$ and $C$.

Table 4: $t \in 2 C$.

\begin{tabular}{|c|c|c|c|c|c|c|}
\hline$C$ & $|\mathcal{O}|$ & $d$ & $c_{1}$ & $c_{2}$ & $c_{3}$ & $c_{4}$ \\
\hline \multirow[t]{2}{*}{$2 A$} & 7920 & 8 & 24 & 31 & 31 & 40 \\
\hline & 495 & 10 & 792 & 495 & 495 & 264 \\
\hline $2 B$ & 25344 & 6 & 32 & 15 & 15 & 0 \\
\hline \multirow{3}{*}{$2 C$} & 47520 & 6 & 24 & 31 & 31 & 40 \\
\hline & 12672 & 8 & 32 & 15 & 15 & 0 \\
\hline & 792 & 10 & 792 & 495 & 495 & 264 \\
\hline $3 A$ & 2048 & 0 & 0 & 0 & 0 & 0 \\
\hline \multirow[t]{2}{*}{$3 B$} & 270336 & 6 & 0 & 0 & 0 & 1 \\
\hline & 112640 & 6 & 0 & 0 & 9 & 6 \\
\hline $3 C$ & 901120 & 3 & 0 & 0 & 0 & 0 \\
\hline $3 D$ & 2027520 & 4 & 0 & 0 & 0 & 0 \\
\hline \multirow[t]{2}{*}{$4 A$} & 126720 & 4 & 0 & 0 & 7 & 0 \\
\hline & 15840 & 6 & 48 & 8 & 63 & 8 \\
\hline \multirow[t]{5}{*}{$4 B$} & 1520640 & 4 & 0 & 0 & 3 & 0 \\
\hline & 380160 & 4 & 0 & 0 & 7 & 0 \\
\hline & 190080 & 6 & 24 & 24 & 15 & 0 \\
\hline & 95040 & 6 & 32 & 16 & 7 & 8 \\
\hline & 95040 & 6 & 48 & 8 & 63 & 8 \\
\hline \multirow[t]{5}{*}{$4 C$} & 1520640 & 5 & 0 & 0 & 3 & 4 \\
\hline & 760320 & 6 & 0 & 0 & 3 & 0 \\
\hline & 380160 & 6 & 32 & 24 & 47 & 24 \\
\hline & 380160 & 6 & 0 & 0 & 7 & 8 \\
\hline & 190080 & 6 & 36 & 20 & 43 & 28 \\
\hline \multirow[t]{3}{*}{$4 D$} & 1520640 & 4 & 0 & 0 & 3 & 4 \\
\hline & 253440 & 6 & 0 & 0 & 7 & 8 \\
\hline & 253440 & 6 & 36 & 20 & 43 & 28 \\
\hline
\end{tabular}


Involutions in Conway's largest simple group

Table 4: $t \in 2 C$, continued from the previous page.

\begin{tabular}{|c|c|c|c|c|c|c|}
\hline$C$ & $|\mathcal{O}|$ & $d$ & $c_{1}$ & $c_{2}$ & $c_{3}$ & $c_{4}$ \\
\hline \multirow{7}{*}{$4 F$} & 190080 & 6 & 32 & 16 & 7 & 8 \\
\hline & 190080 & 6 & 32 & 24 & 47 & 24 \\
\hline & 95040 & 6 & 32 & 24 & 47 & 24 \\
\hline & 3041280 & 4 & 0 & 0 & 1 & 2 \\
\hline & 3041280 & 4 & 0 & 0 & 3 & 0 \\
\hline & 506880 & 6 & 30 & 18 & 9 & 6 \\
\hline & 506880 & 6 & 0 & 0 & 15 & 0 \\
\hline $5 A$ & 1622016 & 0 & 0 & 0 & 0 & 0 \\
\hline \multirow[t]{2}{*}{$5 B$} & 12165120 & 4 & 0 & 0 & 0 & 0 \\
\hline & 3244032 & 4 & 0 & 0 & 0 & 1 \\
\hline $5 C$ & 19464192 & 2 & 0 & 0 & 0 & 0 \\
\hline $6 A$ & 1013760 & 0 & 0 & 0 & 0 & 0 \\
\hline $6 B$ & 1622016 & 0 & 0 & 0 & 0 & 0 \\
\hline \multirow[t]{2}{*}{$6 C$} & 4055040 & 3 & 0 & 0 & 0 & 1 \\
\hline & 4055040 & 3 & 0 & 0 & 3 & 4 \\
\hline $6 D$ & 8110080 & 3 & 0 & 0 & 0 & 0 \\
\hline \multirow[t]{5}{*}{$6 E$} & 12165120 & 4 & 0 & 0 & 0 & 1 \\
\hline & 4055040 & 4 & 0 & 0 & 0 & 1 \\
\hline & 4055040 & 4 & 0 & 0 & 1 & 2 \\
\hline & 4055040 & 4 & 0 & 0 & 0 & 1 \\
\hline & 1013760 & 6 & 0 & 0 & 9 & 6 \\
\hline $6 F$ & 32440320 & 2 & 0 & 0 & 0 & 0 \\
\hline \multirow[t]{2}{*}{$6 G$} & 12165120 & 3 & 0 & 0 & 3 & 4 \\
\hline & 12165120 & 3 & 0 & 0 & 0 & 1 \\
\hline $6 H$ & 24330240 & 2 & 0 & 0 & 0 & 0 \\
\hline \multirow[t]{2}{*}{$6 I$} & 12165120 & 2 & 0 & 0 & 0 & 0 \\
\hline & 12165120 & 4 & 0 & 0 & 0 & 0 \\
\hline $7 A$ & 16220160 & 0 & 0 & 0 & 0 & 0 \\
\hline $7 B$ & 64880640 & 3 & 0 & 0 & 0 & 0 \\
\hline \multirow[t]{2}{*}{$8 A$} & 1013760 & 3 & 0 & 0 & 7 & 0 \\
\hline & 1013760 & 3 & 0 & 0 & 0 & 0 \\
\hline \multirow[t]{2}{*}{$8 B$} & 6082560 & 3 & 0 & 0 & 3 & 4 \\
\hline & 6082560 & 3 & 0 & 0 & 0 & 0 \\
\hline \multirow[t]{3}{*}{$8 C$} & 24330240 & 2 & 0 & 0 & 1 & 0 \\
\hline & 6082560 & 3 & 0 & 0 & 0 & 0 \\
\hline & 6082560 & 3 & 0 & 0 & 7 & 0 \\
\hline \multirow[t]{2}{*}{$8 D$} & 24330240 & 2 & 0 & 0 & 1 & 0 \\
\hline & 6082560 & 4 & 0 & 0 & 3 & 0 \\
\hline
\end{tabular}


Involutions in Conway's largest simple group

Table 4: $t \in 2 C$, continued from the previous page.

\begin{tabular}{|c|c|c|c|c|c|c|}
\hline$C$ & $|\mathcal{O}|$ & $d$ & $c_{1}$ & $c_{2}$ & $c_{3}$ & $c_{4}$ \\
\hline \multirow{10}{*}{$8 E$} & 6082560 & 3 & 0 & 0 & 0 & 0 \\
\hline & 6082560 & 3 & 0 & 0 & 0 & 0 \\
\hline & 6082560 & 3 & 0 & 0 & 7 & 0 \\
\hline & 6082560 & 3 & 0 & 0 & 7 & 0 \\
\hline & 24330240 & 4 & 0 & 0 & 0 & 0 \\
\hline & 24330240 & 4 & 0 & 0 & 1 & 0 \\
\hline & 12165120 & 3 & 0 & 0 & 1 & 0 \\
\hline & 12165120 & 3 & 0 & 0 & 3 & 4 \\
\hline & 6082560 & 4 & 0 & 0 & 1 & 0 \\
\hline & 6082560 & 4 & 0 & 0 & 1 & 2 \\
\hline $9 A$ & 64880640 & 0 & 0 & 0 & 0 & 0 \\
\hline \multirow[t]{2}{*}{$9 B$} & 64880640 & 1 & 0 & 0 & 0 & 0 \\
\hline & 64880640 & 1 & 0 & 0 & 0 & 0 \\
\hline \multirow[t]{2}{*}{$9 C$} & 64880640 & 2 & 0 & 0 & 0 & 0 \\
\hline & 64880640 & 2 & 0 & 0 & 0 & 0 \\
\hline $10 A$ & 24330240 & 0 & 0 & 0 & 0 & 0 \\
\hline \multirow[t]{4}{*}{$10 B$} & 24330240 & 0 & 0 & 0 & 0 & 0 \\
\hline & 24330240 & 0 & 0 & 0 & 0 & 0 \\
\hline & 1622016 & 0 & 0 & 0 & 0 & 0 \\
\hline & 1622016 & 0 & 0 & 0 & 0 & 0 \\
\hline \multirow[t]{2}{*}{$10 C$} & 24330240 & 2 & 0 & 0 & 0 & 0 \\
\hline & 24330240 & 2 & 0 & 0 & 0 & 0 \\
\hline \multirow[t]{2}{*}{$10 D$} & 48660480 & 2 & 0 & 0 & 0 & 0 \\
\hline & 48660480 & 2 & 0 & 0 & 0 & 1 \\
\hline $10 E$ & 97320960 & 2 & 0 & 0 & 0 & 0 \\
\hline \multirow[t]{4}{*}{$10 F$} & 48660480 & 2 & 0 & 0 & 0 & 1 \\
\hline & 24330240 & 4 & 0 & 0 & 0 & 0 \\
\hline & 12165120 & 2 & 0 & 0 & 0 & 0 \\
\hline & 12165120 & 2 & 0 & 0 & 0 & 0 \\
\hline $11 A$ & 194641920 & 2 & 0 & 0 & 0 & 0 \\
\hline \multirow[t]{3}{*}{$12 A$} & 6082560 & 0 & 0 & 0 & 0 & 0 \\
\hline & 1013760 & 0 & 0 & 0 & 0 & 0 \\
\hline & 1013760 & 0 & 0 & 0 & 0 & 0 \\
\hline $12 B$ & 24330240 & 0 & 0 & 0 & 0 & 0 \\
\hline $12 C$ & 24330240 & 0 & 0 & 0 & 0 & 0 \\
\hline \multirow[t]{2}{*}{$12 D$} & 32440320 & 1 & 0 & 0 & 0 & 0 \\
\hline & 32440320 & 1 & 0 & 0 & 0 & 0 \\
\hline $12 E$ & 48660480 & 2 & 0 & 0 & 0 & 0 \\
\hline
\end{tabular}


Involutions in Conway's largest simple group

Table 4: $t \in 2 C$, continued from the previous page.

\begin{tabular}{|c|c|c|c|c|c|c|}
\hline$C$ & $|\mathcal{O}|$ & $d$ & $c_{1}$ & $c_{2}$ & $c_{3}$ & $c_{4}$ \\
\hline \multirow{6}{*}{$12 G$} & 16220160 & 2 & 0 & 0 & 1 & 0 \\
\hline & 8110080 & 4 & 0 & 0 & 3 & 0 \\
\hline & 24330240 & 2 & 0 & 0 & 0 & 0 \\
\hline & 24330240 & 2 & 0 & 0 & 1 & 0 \\
\hline & 24330240 & 2 & 0 & 0 & 1 & 0 \\
\hline & 24330240 & 2 & 0 & 0 & 0 & 0 \\
\hline \multirow[t]{2}{*}{$12 H$} & 97320960 & 2 & 0 & 0 & 0 & 0 \\
\hline & 48660480 & 3 & 0 & 0 & 0 & 0 \\
\hline \multirow[t]{4}{*}{$12 I$} & 48660480 & 2 & 0 & 0 & 0 & 1 \\
\hline & 48660480 & 2 & 0 & 0 & 1 & 0 \\
\hline & 24330240 & 3 & 0 & 0 & 0 & 0 \\
\hline & 24330240 & 3 & 0 & 0 & 5 & 2 \\
\hline \multirow[t]{4}{*}{$12 J$} & 48660480 & 2 & 0 & 0 & 0 & 0 \\
\hline & 48660480 & 2 & 0 & 0 & 0 & 1 \\
\hline & 8110080 & 4 & 0 & 0 & 1 & 0 \\
\hline & 8110080 & 4 & 0 & 0 & 1 & 2 \\
\hline \multirow[t]{2}{*}{$12 K$} & 97320960 & 1 & 0 & 0 & 0 & 0 \\
\hline & 97320960 & 1 & 0 & 0 & 0 & 0 \\
\hline \multirow[t]{2}{*}{$12 M$} & 48660480 & 2 & 0 & 0 & 0 & 0 \\
\hline & 48660480 & 2 & 0 & 0 & 0 & 0 \\
\hline $13 A$ & 97320960 & 0 & 0 & 0 & 0 & 0 \\
\hline $14 A$ & 97320960 & 0 & 0 & 0 & 0 & 0 \\
\hline $14 B$ & 194641920 & 2 & 0 & 0 & 0 & 0 \\
\hline $15 A$ & 38928384 & 0 & 0 & 0 & 0 & 0 \\
\hline $15 B$ & 97320960 & 0 & 0 & 0 & 0 & 0 \\
\hline $15 C$ & 97320960 & 0 & 0 & 0 & 0 & 0 \\
\hline \multirow[t]{2}{*}{$15 D$} & 194641920 & 2 & 0 & 0 & 0 & 0 \\
\hline & 64880640 & 2 & 0 & 0 & 0 & 0 \\
\hline \multirow[t]{2}{*}{$15 E$} & 194641920 & 1 & 0 & 0 & 0 & 0 \\
\hline & 194641920 & 1 & 0 & 0 & 0 & 0 \\
\hline \multirow[t]{2}{*}{$16 B$} & 97320960 & 2 & 0 & 1 & 0 & 0 \\
\hline & 97320960 & 2 & 0 & 0 & 0 & 0 \\
\hline $18 A$ & 194641920 & 0 & 0 & 0 & 0 & 0 \\
\hline \multirow[t]{2}{*}{$18 B$} & 194641920 & 1 & 0 & 0 & 0 & 0 \\
\hline & 194641920 & 1 & 0 & 0 & 0 & 0 \\
\hline \multirow[t]{2}{*}{$18 C$} & 194641920 & 1 & 0 & 0 & 0 & 0 \\
\hline & 194641920 & 1 & 0 & 0 & 0 & 0 \\
\hline $20 A$ & 97320960 & 0 & 0 & 0 & 0 & 0 \\
\hline
\end{tabular}


Involutions in Conway's largest simple group

Table 4: $t \in 2 C$, continued from the previous page.

\begin{tabular}{|c|c|c|c|c|c|c|}
\hline$C$ & $|\mathcal{O}|$ & $d$ & $c_{1}$ & $c_{2}$ & $c_{3}$ & $c_{4}$ \\
\hline \multirow[t]{2}{*}{$20 B$} & 97320960 & 2 & 0 & 0 & 0 & 0 \\
\hline & 97320960 & 2 & 0 & 0 & 0 & 0 \\
\hline \multirow[t]{2}{*}{$20 C$} & 194641920 & 1 & 0 & 0 & 0 & 0 \\
\hline & 194641920 & 1 & 0 & 0 & 0 & 0 \\
\hline $21 A$ & 97320960 & 0 & 0 & 0 & 0 & 0 \\
\hline $21 B$ & 129761280 & 0 & 0 & 0 & 0 & 0 \\
\hline $21 C$ & 389283840 & 1 & 0 & 0 & 0 & 0 \\
\hline \multirow[t]{2}{*}{$24 B$} & 97320960 & 0 & 0 & 0 & 0 & 0 \\
\hline & 97320960 & 0 & 0 & 0 & 0 & 0 \\
\hline \multirow[t]{2}{*}{$24 C$} & 32440320 & 2 & 0 & 0 & 1 & 0 \\
\hline & 32440320 & 2 & 0 & 0 & 0 & 0 \\
\hline \multirow[t]{2}{*}{$24 E$} & 97320960 & 2 & 0 & 0 & 0 & 0 \\
\hline & 97320960 & 2 & 0 & 0 & 1 & 0 \\
\hline \multirow[t]{2}{*}{$24 F$} & 194641920 & 2 & 0 & 0 & 0 & 0 \\
\hline & 194641920 & 2 & 0 & 0 & 0 & 0 \\
\hline \multirow[t]{2}{*}{$26 A$} & 97320960 & 0 & 0 & 0 & 0 & 0 \\
\hline & 97320960 & 0 & 0 & 0 & 0 & 0 \\
\hline \multirow[t]{2}{*}{$28 A$} & 194641920 & 1 & 0 & 0 & 0 & 0 \\
\hline & 194641920 & 1 & 0 & 0 & 0 & 0 \\
\hline $30 A$ & 194641920 & 0 & 0 & 0 & 0 & 0 \\
\hline \multirow[t]{2}{*}{$30 B$} & 97320960 & 0 & 0 & 0 & 0 & 0 \\
\hline & 97320960 & 0 & 0 & 0 & 0 & 0 \\
\hline \multirow[t]{2}{*}{$30 C$} & 97320960 & 0 & 0 & 0 & 0 & 0 \\
\hline & 97320960 & 0 & 0 & 0 & 0 & 0 \\
\hline \multirow[t]{2}{*}{$30 D$} & 194641920 & 1 & 0 & 0 & 0 & 0 \\
\hline & 194641920 & 1 & 0 & 0 & 0 & 0 \\
\hline \multirow[t]{2}{*}{$30 E$} & 194641920 & 1 & 0 & 0 & 0 & 0 \\
\hline & 194641920 & 1 & 0 & 0 & 0 & 0 \\
\hline $33 A$ & 389283840 & 0 & 0 & 0 & 0 & 0 \\
\hline $35 A$ & 389283840 & 0 & 0 & 0 & 0 & 0 \\
\hline \multirow[t]{2}{*}{$36 A$} & 194641920 & 0 & 0 & 0 & 0 & 0 \\
\hline & 194641920 & 0 & 0 & 0 & 0 & 0 \\
\hline \multirow[t]{2}{*}{$42 A$} & 97320960 & 0 & 0 & 0 & 0 & 0 \\
\hline & 97320960 & 0 & 0 & 0 & 0 & 0 \\
\hline \multirow[t]{2}{*}{$60 A$} & 194641920 & 0 & 0 & 0 & 0 & 0 \\
\hline & 194641920 & 0 & 0 & 0 & 0 & 0 \\
\hline
\end{tabular}




\subsection{Suborbit matrix representatives}

In Appendix B, we give matrix representatives for each suborbit. There are three main folders in the file, each labelled 'Co1_X_reps' for $\mathrm{X}$ in $\{2 A, 2 B, 2 C\}$, and corresponding to suborbit representatives for the appropriate class. In each case, the representatives are dimension-24 matrices over the Galois field $G F(2)$, and are provided in three formats: GAP [14], Magma [2] and MeatAxe (see [16] for more information on these formats). More-detailed information about the structure of the folders and the presentation of the data can be found in the 'README' file in the top-level directory of the folder.

In both the GAP and MAGMA cases, all the representatives for a given class are contained in one file, and then systematically labelled in accordance with the tables given in Section 2. Indeed, we specify a matrix $t$, and then each representative $x$ has a name of the form ' $x C_{-} i$ ', where $C$ is the ATLAs [7] name of the $G$-conjugacy class containing $t x$, and $i$ indicates that $x$ is a representative for the $i$ th suborbit, with respect to the appropriate table in Section 2 . For example, after loading the 'Co1_2C_reps_MAGMA' file into the computer algebra package MaGma, the element stored as ' $x 4 B \_3$ ' corresponds to the suborbit representative $x$ whose product $t x$ is in class $4 B$, and which lies in a suborbit of size 190080 .

For each representative in the MeatAxe case, there is a file 'Meataxe_ $X \_S$ ' for each $X$ in $\{2 A, 2 B, 2 C\}$ and each corresponding non-zero structure constant $S$. Within each file, the $i$ th matrix given corresponds to the $i$ th suborbit with respect to the tables in Section 2. So to obtain the representative in the example above, one would open the file 'Meataxe_2C_4B', and take the third matrix down as listed.

In each of the cases $t \in\{2 A, 2 B, 2 C\}$, we also provide generators for the centralizer $C_{G}(t)$ and some of its subgroups. For $t \in 2 A$, we have $C_{G}(t)=N^{\cdot} H \cong 2_{+}^{1+8} \cdot O_{8}^{+}(2)$, and we provide generators for both the full centralizer and the extra-special group $N$. When $t \in 2 B$, we have $C_{G}(t) \cong\left(2^{2} \times G_{2}(4)\right): 2$, which has subgroup $D=F \times L \cong 2^{2} \times G_{2}(4)$, of index 2. We provide generators for the full centralizer, for $D$, and for its subgroups $F$ and $L$. Finally, if $t \in 2 C$, we have $C_{G}(t)=N: M \cong 2^{11}: M_{12}: 2$, and again we give generators for both the full centralizer and the elementary abelian group $N$.

Appendix A. Data that may facilitate faster identification of suborbits

\begin{tabular}{l|cll}
\hline$g \in C$ & $\operatorname{dim}\left(C_{V}(g)\right)$ & $\operatorname{dim}\left(C_{V}\left(g^{i}\right)\right)$ & $\begin{array}{l}\text { Additional information } \\
\text { used in Section } 2\end{array}$ \\
\hline $1 A$ & 24 & - & - \\
$2 A$ & 16 & - & - \\
$2 B$ & 12 & - & Generate $C_{G}(g)$ using Bray's method [3]. \\
& & & Elements of order 13 in $C_{G}(g)$ \\
& & & indicate that $g \in 2 B$. \\
$2 C$ & 12 & - & Generate $C_{G}(g)$ using Bray's method [3]. \\
& & & Elements of order 11 in $C_{G}(g)$ \\
$3 A$ & 0 & - & indicate that $g \in 2 C$. \\
$3 B$ & 12 & - & - \\
\hline
\end{tabular}


Appendix A, continued from the previous page.

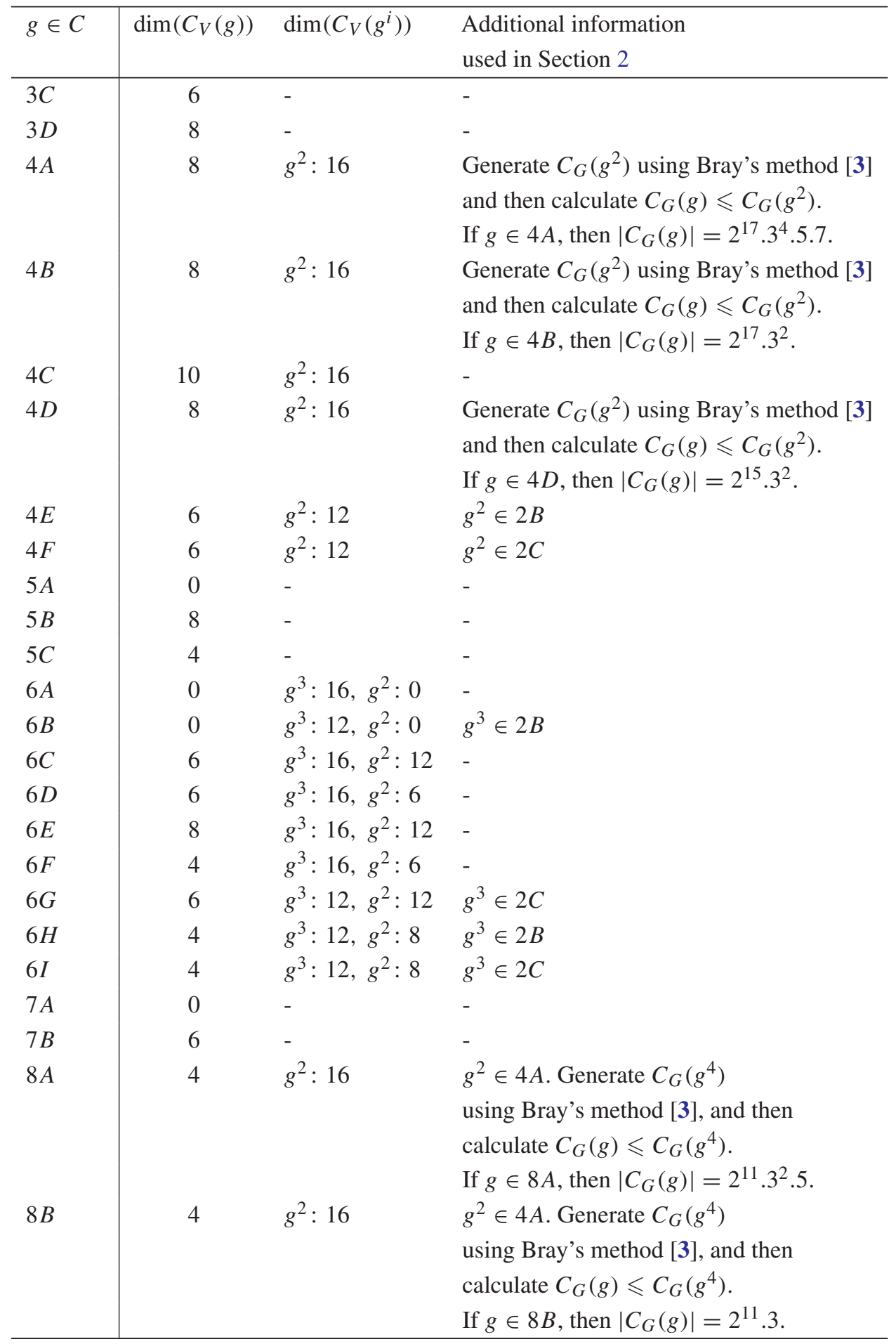


Appendix A, continued from the previous page.

\begin{tabular}{|c|c|c|c|}
\hline$g \in C$ & $\operatorname{dim}\left(C_{V}(g)\right)$ & $\operatorname{dim}\left(C_{V}\left(g^{i}\right)\right)$ & $\begin{array}{l}\text { Additional information } \\
\text { used in Section } 2\end{array}$ \\
\hline $8 C$ & 4 & $g^{2}: 16$ & $\begin{array}{l}g^{2} \in 4 B \text {. Generate } C_{G}\left(g^{4}\right) \\
\text { using Bray's method [3], and then } \\
\text { calculate } C_{G}(g) \leqslant C_{G}\left(g^{4}\right) \text {. } \\
\text { If } g \in 8 C \text {, then }\left|C_{G}(g)\right|=2^{11} .3 \text {. }\end{array}$ \\
\hline $8 D$ & 4 & $g^{2}: 16$ & $\begin{array}{l}g^{2} \in 4 B \text {. Generate } C_{G}\left(g^{4}\right) \\
\text { using Bray's method [3], and then } \\
\text { calculate } C_{G}(g) \leqslant C_{G}\left(g^{4}\right) \text {. } \\
\text { If } g \in 8 D \text {, then }\left|C_{G}(g)\right|=2^{11} \text {. }\end{array}$ \\
\hline $8 E$ & 6 & $g^{2}: 16$ & $g^{2} \in 4 C$ \\
\hline $8 F$ & 4 & $g^{2}: 16$ & $g^{2} \in 4 D$ \\
\hline $9 A$ & 0 & $g^{3}: 6$ & - \\
\hline $9 B$ & 2 & $g^{3}: 6$ & - \\
\hline $9 C$ & 4 & $g^{3}: 6$ & - \\
\hline $10 A$ & 0 & $g^{5}: 16, g^{2}: 0$ & - \\
\hline $10 B$ & 0 & $g^{5}: 12, g^{2}: 0$ & $g^{5} \in 2 B$ \\
\hline $10 C$ & 4 & $g^{5}: 12, g^{2}: 8$ & $g^{5} \in 2 B$ \\
\hline $10 D$ & 4 & $g^{5}: 16, g^{2}: 8$ & - \\
\hline $10 E$ & 4 & $g^{5}: 16, g^{2}: 4$ & - \\
\hline $10 F$ & 4 & $g^{5}: 12, g^{2}: 8$ & $g^{5} \in 2 C$ \\
\hline $11 A$ & 4 & - & - \\
\hline $12 A$ & 0 & $g^{3}: 8, g^{2}: 0$ & $g^{3} \in 4 A, g^{2} \in 6 A$ \\
\hline $12 B$ & 0 & $g^{3}: 8, g^{2}: 0$ & $g^{3} \in 4 B, g^{2} \in 6 A$ \\
\hline $12 C$ & 0 & $g^{3}: 8, g^{2}: 0$ & $g^{3} \in 4 D, g^{2} \in 6 A$ \\
\hline $12 D$ & 2 & $g^{3}: 8, g^{2}: 4$ & $g^{3} \in 4 A, g^{2} \in 6 F$ \\
\hline $12 E$ & 4 & $g^{3}: 8, g^{2}: 8$ & $g^{3} \in 4 A, g^{2} \in 6 E$ \\
\hline $12 F$ & 0 & $g^{3}: 6, g^{2}: 0$ & $g^{3} \in 4 E, g^{2} \in 6 B$ \\
\hline $12 G$ & 4 & $g^{3}: 8, g^{2}: 8$ & $g^{3} \in 4 B, g^{2} \in 6 E$ \\
\hline $12 H$ & 4 & $g^{3}: 10, g^{2}: 6$ & $g^{3} \in 4 C, g^{2} \in 6 D$ \\
\hline $12 I$ & 4 & $g^{3}: 10, g^{2}: 8$ & $g^{3} \in 4 C, g^{2} \in 6 E$ \\
\hline $12 J$ & 4 & $g^{3}: 8, g^{2}: 8$ & $g^{3} \in 4 D, g^{2} \in 6 E$ \\
\hline $12 K$ & 2 & $g^{3}: 8, g^{2}: 4$ & $g^{3} \in 4 B, g^{2} \in 6 F$ \\
\hline $12 L$ & 2 & $g^{3}: 6, g^{2}: 4$ & $g^{3} \in 4 E, g^{2} \in 6 H$ \\
\hline $12 M$ & 2 & $g^{3}: 6, g^{2}: 4$ & $g^{3} \in 4 F, g^{2} \in 6 I$ \\
\hline $13 A$ & 0 & - & - \\
\hline $14 A$ & 0 & $g^{7}: 12, g^{2}: 0$ & - \\
\hline $14 B$ & 4 & $g^{7}: 16, g^{2}: 6$ & - \\
\hline
\end{tabular}


Involutions in Conway's largest simple group

Appendix A, continued from the previous page.

\begin{tabular}{|c|c|c|c|}
\hline$g \in C$ & $\operatorname{dim}\left(C_{V}(g)\right)$ & $\operatorname{dim}\left(C_{V}\left(g^{i}\right)\right)$ & $\begin{array}{l}\text { Additional information } \\
\text { used in Section } 2\end{array}$ \\
\hline $15 A$ & 0 & $g^{5}: 0, g^{3}: 0$ & - \\
\hline $15 B$ & 0 & $g^{5}: 0, g^{3}: 8$ & - \\
\hline $15 C$ & 0 & $g^{5}: 8, g^{3}: 0$ & - \\
\hline $15 D$ & 4 & $g^{5}: 12, g^{3}: 8$ & - \\
\hline $15 E$ & 2 & $g^{5}: 6, g^{3}: 4$ & - \\
\hline $16 A$ & 0 & $g^{2}: 4$ & $g^{2} \in 8 C$ \\
\hline $16 B$ & 0 & $g^{2}: 4$ & $g^{2} \in 8 D$ \\
\hline $18 A$ & 0 & $g^{3}: 4, g^{2}: 0$ & - \\
\hline $18 B$ & 2 & $g^{3}: 4, g^{2}: 2$ & - \\
\hline $18 C$ & 2 & $g^{3}: 4, g^{2}: 4$ & - \\
\hline $20 A$ & 0 & $g^{5}: 8, g^{2}: 0$ & - \\
\hline $20 B$ & 2 & $g^{5}: 6, g^{2}: 4$ & - \\
\hline $20 C$ & 2 & $g^{5}: 10, g^{2}: 4$ & - \\
\hline $21 A$ & 0 & $g^{7}: 0, g^{3}: 0$ & - \\
\hline $21 B$ & 0 & $g^{7}: 12, g^{3}: 0$ & - \\
\hline $21 C$ & 2 & $g^{7}: 8, g^{3}: 6$ & - \\
\hline $23 A$ & 2 & - & - \\
\hline $23 B * *$ & 2 & - & - \\
\hline $24 A$ & 0 & $g^{3}: 4, g^{2}: 0$ & $g^{3} \in 8 A, g^{2} \in 12 A$ \\
\hline $24 B$ & 0 & $g^{3}: 4, g^{2}: 0$ & $g^{3} \in 8 C, g^{2} \in 12 B$ \\
\hline $24 C$ & 2 & $g^{3}: 4, g^{2}: 4$ & $g^{3} \in 8 A, g^{2} \in 12 E$ \\
\hline $24 D$ & 0 & $g^{3}: 4, g^{2}: 0$ & $g^{3} \in 8 F, g^{2} \in 12 C$ \\
\hline $24 E$ & 2 & $g^{3}: 4, g^{2}: 4$ & $g^{3} \in 8 B, g^{2} \in 12 E$ \\
\hline $24 F$ & 2 & $g^{3}: 6, g^{2}: 4$ & $g^{3} \in 8 E, g^{2} \in 12 H$ \\
\hline $26 A$ & 0 & $g^{13}: 12, g^{2}: 0$ & - \\
\hline $28 A$ & 2 & $g^{7}: 8, g^{2}: 4$ & - \\
\hline $28 B$ & 0 & $g^{7}: 6, g^{2}: 0$ & - \\
\hline $30 A$ & 0 & $\begin{array}{l}g^{5}: 0, g^{3}: 0 \\
g^{2}: 0\end{array}$ & - \\
\hline $30 B$ & 0 & $\begin{array}{l}g^{5}: 0, g^{3}: 4 \\
g^{2}: 0\end{array}$ & - \\
\hline $30 C$ & 0 & $\begin{array}{l}g^{5}: 4, g^{3}: 0 \\
g^{2}: 0\end{array}$ & - \\
\hline $30 D$ & 2 & $\begin{array}{l}g^{5}: 6, g^{3}: 4 \\
g^{2}: 4\end{array}$ & - \\
\hline
\end{tabular}


Appendix A, continued from the previous page.

\begin{tabular}{l|cll}
\hline$g \in C$ & $\operatorname{dim}\left(C_{V}(g)\right)$ & $\operatorname{dim}\left(C_{V}\left(g^{i}\right)\right)$ & $\begin{array}{l}\text { Additional information } \\
\text { used in Section } 2\end{array}$ \\
\hline $30 E$ & 2 & $g^{5}: 6 g^{3}: 4$, & - \\
& & $g^{2}: 2$ & \\
& & $g^{11}: 0, g^{3}: 4$ & - \\
$33 A$ & 0 & $g^{7}: 0, g^{5}: 0$ & - \\
$35 A$ & 0 & $g^{3}: 2, g^{2}: 0$ & - \\
$36 A$ & 0 & $g^{13}: 0, g^{3}: 0$ & - \\
$39 A$ & 0 & $g^{13}: 0, g^{3}: 0$ & - \\
$39 B * *$ & 0 & $g^{5}: 4, g^{2}: 0$ & - \\
$40 A$ & 0 & $g^{7}: 0, g^{3}: 0$, & - \\
$42 A$ & 0 & $g^{2}: 0$ \\
& & $g^{5}: 0, g^{3}: 0$, & - \\
$60 A$ & 0 & $g^{2}: 0$ & \\
\end{tabular}

Appendix B. Matrix representatives for each suborbit

The files mentioned in Section 2.4 are available at

http://www. lms.ac.uk/jcm/7/lms2003-030/appendix-b.

\section{References}

1. C. Bates and P. Rowley, 'Centralizers of real elements in finite groups, Arch. Math., to appear. 338

2. W. Bosma, J. Cannon and C. Playoust, 'The Magma algebra system I: The user language', J. Symbolic Comput. 24 (1997) 235-265. 338, 346

3. J. BRAY, 'An improved method for generating the centralizer of an involution', Arch. Math. 74 (2000) 241-245. 338, 346, 347, 348

4. T. Breuer and G. Pfeiffer, 'Finding possible permutation characters', J . Symbolic Comput. 26 (1998) 343-354. 337

5. J. H. Conway, 'A simple construction for the Fischer-Griess Monster group', Invent. Math. 79 (1985) 513-540. 337

6. J. H. Conway, 'Three lectures on exceptional groups', Finite simple groups (Proc. Instructional Conf., Oxford, 1969) (Academic Press, London, 1971) 215-247. 337

7. J. H. Conway, R. T. Curtis, S. P. Norton, R. A. Parker and R .A. Wilson, Atlas of finite groups (Clarendon Press, Oxford, 1985). 337, 338, 346

8. J. H. Conway and N. J. A. Sloane, Sphere packings, lattices and groups, 3rd edn., with additional contributions by E. Bannai, R. E. Borcherds, J. Leech, S. P. Norton, A. M. Odlyzko, R. A. Parker, L. Queen and B. B. Venkov, Grund. Math. Wiss. [Fundamental Principles of Mathematical Sciences] 290 (Springer, New York, 1999). 337

9. R. T. Curtis, On subgroups of .0. I. Lattice stabilizers. J. Algebra 27 (1973) 549-573. 337 
10. R. T. Curtis, On subgroups of .0. II. Local structure. J. Algebra 63 (1980) 413-434. 337

11. D. Gorenstein, Finite groups 2nd edn (Chelsea Publishing Co., New York, 1980). 338

12. R. L. GrIESS, JR., 'The friendly giant', Invent. Math. 69 (1982) 1-102. 337

13. K. HARAdA and M.-L. LANG, 'Some elliptic curves arising from the Leech lattice', J. Algebra 125 (1989) 298-310. 337

14. The GAP Group, GAP - groups, algorithms, and programming, Version 4.3, 2002, http: / /www . gap-system.org. 337, 338, 346

15. R. A. Wilson, 'The maximal subgroups of Conway's group $\mathrm{Co}_{1}$ ', J. Algebra 85 (1983) 144-165. 337

16. R. Wilson, P. Walsh, J. Tripp, I. Suleiman, S. Rogers, R. Parker, S. Norton, S. Linton and J. Bray, 'Atlas of finite group representations', http: //web.mat.bham.ac.uk/atlas/v2 . 0/. 338, 339, 346

Chris Bates c.bates@postgrad.manchester.ac.uk

http://personalpages.umist.ac.uk/postgrad/c.bates

Peter Rowley peter.j.rowley@manchester.ac.uk

http://www.ma.umist.ac.uk/pjr

School of Mathematics

The University of Manchester

P O Box 88

Sackville Street

Manchester M60 1QD

United Kingdom 